\title{
Contribution of alginate and levan production to biofilm formation by Pseudomonas syringae
}

Correspondence

Matthias S. Ullrich

m.ullrich@iu-bremen.de

Received 28 January 2006

Revised 21 June 2006

Accepted 27 June 2006

\author{
Heike Laue, ${ }^{1} \dagger$ Alexander Schenk, ${ }^{2}$ Hongqiao Li, ${ }^{2}$ Lotte Lambertsen, ${ }^{1} \ddagger$ \\ Thomas R. Neu, ${ }^{3}$ Søren Molin ${ }^{1}$ and Matthias S. Ullrich ${ }^{2}$
${ }^{1}$ Molecular Microbial Ecology Group, BioCentrum-DTU, Technical University of Denmark, DK-2800 Lyngby, Denmark Bremen, Germany
${ }^{3}$ Department of River Ecology, UFZ Centre for Environmental Research, D-39114 Magdeburg, Germany \\ ${ }^{2}$ School of Engineering and Sciences, International University Bremen, Campusring 1, D-28759
}

Exopolysaccharides (EPSs) play important roles in the attachment of bacterial cells to a surface and/or in building and maintaining the three-dimensional, complex structure of bacterial biofilms. To elucidate the spatial distribution and function of the EPSs levan and alginate during biofilm formation, biofilms of Pseudomonas syringae strains with different EPS patterns were compared. The mucoid strain PG4180.muc, which produces levan and alginate, and its levan- and/or alginate-deficient derivatives all formed biofilms in the wells of microtitre plates and in flow chambers. Confocal laser scanning microscopy with fluorescently labelled lectins was applied to investigate the spatial distribution of levan and an additional as yet unknown EPS in flow-chamber biofilms. Concanavalin A (ConA) bound specifically to levan and accumulated in cell-depleted voids in the centres of microcolonies and in blebs. No binding of ConA was observed in biofilms of the levan-deficient mutants or in wild-type biofilms grown in the absence of sucrose as confirmed by an enzyme-linked lectin-sorbent assay using peroxidase-linked ConA. Time-course studies revealed that expression of the levan-forming enzyme, levansucrase, occurred mainly during early exponential growth of both planktonic and sessile cells. Thus, accumulation of levan in biofilm voids hints to a function as a nutrient storage source for later stages of biofilm development. The presence of a third EPS besides levan and alginate was indicated by binding of the lectin from Naja mossambica to a fibrous structure in biofilms of all $P$. syringae derivatives. Production of the as yet uncharacterized additional EPS might be more important for biofilm formation than the syntheses of levan and alginate.

\section{INTRODUCTION}

Biofilms are defined as communities of microbial cells growing on a surface and embedded in a self-synthesized matrix composed of extracellular polymeric substances. The major components of these are exopolysaccharides (EPSs) (Sutherland, 2001), but DNA (Whitchurch et al., 2002), proteins and lipids (Wingender et al., 1999) can make up a significant proportion of the matrix in biofilms. This matrix is considered to be the key component that determines the physico-chemical and biological properties of biofilms

tPresent address: Arpida Ltd, CH-4142 Muenchenstein, Switzerland. łPresent address: Statens Serum Institut, DK-2100 Copenhagen, Denmark.

Abbreviations: ABTS, 2,2'-azino-bis(3-ethylbenzthiazoline-6-sulfonic acid); CLSM, confocal laser scanning microscopy; ConA, Concanavalin A; ELLA, enzyme-linked lectin-sorbent assay; EPS, exopolysaccharide.
(Wolfaardt et al., 1999). EPSs have been shown to be required for the initial attachment to a surface of Vibrio cholerae (Watnick \& Kolter, 1999) and Staphylococcus epidermidis (McKenney et al., 1998) or for the structural development of mature biofilms like colanic acid in Escherichia coli K-12 biofilms (Danese et al., 2000).

Pseudomonas syringae pv. glycinea is the causal agent of bacterial blight on soybean plants. The bacterium is known to produce two different EPSs, alginate and levan (Osman et al., 1986). Levan is a high-molecular-mass $\beta$-(2,6)-polyfructan with extensive branching through $\beta$ - $(2,1)$-linkages. Its synthesis from sucrose is catalysed by one single, extracellular enzyme, levansucrase (EC 2.4.1.10). Many Gram-negative (e.g. Acetobacter diazotrophicus, P. syringae, Erwinia amylovora; Arrieta et al., 1996; Bogs \& Geider, 2000; Hettwer et al., 1995) and Gram-positive bacteria (e.g. Bacillus subtilis, Streptococcus mutans; Dedonder, 1966; Sato et al., 1984) 
produce levansucrases. Three $l s c$ genes encoding levansucrase (of which only two are expressed) have been identified in $P$. syringae pv. glycinea PG4180 and mutants defective in levan formation were constructed (Li \& Ullrich, 2001).

With the exception of dental biofilms, the role of levan in biofilm formation has not been studied so far. Fructans produced by $S$. mutans are believed to function primarily as extracellular storage compounds that are metabolized during periods of nutrient deprivation (Burne et al., 1996). P. syringae mainly produces levan when sucrose is present. In contrast, alginate has been shown to be produced by this bacterium when it infects plant leaves (Fett \& Dunn, 1989; Osman et al., 1986). Alginate is a co-polymer of $\beta-1,4-$ linked D-mannuronic and L-guluronic acid, which is normally $\mathrm{O}$-acetylated at the 2 and/or 3 position(s) of the D-mannuronate residues (Osman et al., 1986). The arrangement of the alginate structural gene cluster of $P$. syringae is virtually identical to that described for Pseudomonas aeruginosa (Penaloza-Vazquez et al., 1997). Alginate production by $P$. syringae has been associated with increased epiphytic fitness, resistance to desiccation and toxic molecules (Yu et al., 1999), and the induction of watersoaked lesions on infected leaves (Fett \& Dunn, 1989). The algA gene is one of the genes induced during $P$. syringae pv. tomato infection of Arabidopsis thaliana as shown by IVET technology (Boch et al., 2002). Likewise, the algD gene is expressed when $P$. syringae attempts to colonize both susceptible and resistant plant hosts (Keith et al., 2003).

Due to their specific binding to carbohydrates, fluorescently labelled lectins combined with confocal laser scanning microscopy (CLSM) (Lawrence et al., 1998; Mathee et al., 1999; Neu \& Lawrence, 1999; Strathmann et al., 2002) or with enzyme-linked lectin-sorbent assays (ELLAs) have been applied for analysis of EPSs in biofilms (Leriche et al., 2000; Strathmann et al., 2002; Thomas et al., 1997).

The goal of this study was to determine the spatial distribution and function of EPSs during biofilm formation by $P$. syringae using defined alginate- and/or levan-deficient mutants in combination with specific EPS staining. We hypothesized that alginate and levan might have either structure-determining or nutrient storage function(s).

\section{METHODS}

Bacterial strains and growth conditions. Bacterial strains used in this study are described in Table 1. Strain PG4180 produces levan but not alginate due to a nonsense mutation in the key regulatory gene, $\operatorname{alg} T$, as confirmed by nucleotide sequence determination of this gene (A. Schenk, L. M. Keith, C. L. Bender \& M. Ullrich, unpublished data). The mucoid derivative of PG4180, PG4180.muc, emerged spontaneously from a PG4180 culture and is characterized by a reversion of this mutation (L. M. Keith \& C. L. Bender, unpublished data). PG4180.muc produces both alginate and levan. The levan- and alginate-deficient mutant PG4180.M6 had previously been generated from PG4180 as an $l s c B l s c C$ double mutant by marker exchange mutagenesis (Li \& Ullrich, 2001). This mutant could be complemented to a levan-positive phenotype by either $l s c B$ or $l s c C$. The alginate-producing transconjugant PG4180.M6 (pBBR3AXSalgT) carries a plasmid containing the intact alg $T$ gene and its upstream promoter region cloned from strain PG4180.muc (A. Schenk \& M. S. Ullrich, unpublished data). PG4180.M6 (pRLB7.2) represents a transconjugant restored in levan formation by providing a functional $l s c B$ gene in trans. PG4180.muc-GFP was fluorescently tagged with a $g f p$ gene from a mini-Tn7 construct (Klausen et al., 2003; Koch et al., 2001) at an intergenic neutral chromosomal locus. The integration of the $g f p$-containing insert was confirmed by nucleotide sequencing.

Modified FAB medium (Hentzer et al., 2001) supplemented with $10 \mathrm{mM}$ glucose and $10 \mathrm{mM}$ sucrose was used for batch overnight

Table 1. $P$. syringae pv. glycinea strains used in this study

\begin{tabular}{|c|c|c|c|c|}
\hline \multirow[t]{2}{*}{ Strain } & \multicolumn{2}{|c|}{ EPSs produced } & \multirow[t]{2}{*}{ Relevant characteristics ${ }^{\star}$} & \multirow[t]{2}{*}{ Reference } \\
\hline & Levan & Alginate & & \\
\hline PG4180 & + & - & $\begin{array}{l}\text { Wild-type producing negligible amounts of } \\
\text { alginate due to mutation in } \operatorname{alg} T\end{array}$ & Li \& Ullrich (2001) \\
\hline PG4180.M6 & - & - & $\begin{array}{l}l s c B \text { lscC mutant derived from PG4180, } \\
\text { mutation in algT; } \mathrm{Gm}^{\mathrm{r}} \mathrm{Sm} / \mathrm{Sp}^{\mathrm{r}}\end{array}$ & Li \& Ullrich (2001) \\
\hline PG4180.muc-GFP & + & + & $\begin{array}{l}\text { eGFP under control of ribosomal promoter, } \\
\text { integrated using } \mathrm{Tn} 7 \text { transposon; } \mathrm{Gm}^{\mathrm{r}} \mathrm{Cm}^{\mathrm{r}}\end{array}$ & This study \\
\hline PG4180.M6(pRLB7.2) & + & - & $\begin{array}{l}\text { lscB } l s c C \text { mutant, plasmid-encoded } \\
\text { functional } l s c B ; \mathrm{Gm}^{\mathrm{r}} \mathrm{Sm} / \mathrm{Sp}^{\mathrm{r}} \mathrm{Cm}^{\mathrm{r}}\end{array}$ & This study \\
\hline
\end{tabular}

${ }^{\star} \mathrm{Cm}^{\mathrm{r}}$, chloramphenicol resistant; $\mathrm{Gm}^{\mathrm{r}}$, gentamicin-resistant; $\mathrm{Sm} / \mathrm{Sp}^{\mathrm{r}}$, streptomycin and spectinomycin-resistant; Tc ${ }^{\mathrm{r}}$, tetracycline-resistant; $l s c B$ $l s c C$, genes encoding levansucrase. 
cultures grown at $20^{\circ} \mathrm{C}$. Mannitol-glutamate (MG) medium (Keane et al., 1970) agar plates supplemented with $5 \%$ sucrose and incubated at $20^{\circ} \mathrm{C}$ were used to determine levan production of $P$. syringae. The following antibiotics $\left(\mathrm{mg} \mathrm{ml}^{-1}\right)$ were added to the media when needed: gentamicin, 2; streptomycin, 25; tetracycline, 25; chloramphenicol, 25.

Microtitre plate biofilm formation assay. To compare the impact of levan and alginate production on initial biofilm formation, biofilms of $P$. syringae PG4180, PG4180.muc, PG4180.M6, PG4180.M6(pBBR3-AXSalgT) and PG4180.M6 (pRLB7.2) were grown in wells of polystyrene 96 -well microtitre plates at $20^{\circ} \mathrm{C}$ for $48 \mathrm{~h}$ as follows. Overnight cultures of $P$. syringae were diluted to an $\mathrm{OD}_{600}$ of 0.02 in $\mathrm{FAB}$ minimal medium supplemented with $60 \mathrm{mM}$ glucose or $10 \mathrm{mM}$ glucose plus $50 \mathrm{mM}$ sucrose. Subsequently, aliquots of $100 \mu \mathrm{l}$ were placed into the wells of microtitre plates. The amount of surface-attached biofilm was determined by using a modified crystal violet method (O'Toole \& Kolter, 1998) as described elsewhere (Klausen et al., 2003). The biofilm cell-associated dye was measured as its absorption at $590 \mathrm{~nm}$.

ELLA of microtitre plate biofilms. An ELLA was performed according to the procedure described by Leriche et al. (2000). Microtitre plate biofilms of $P$. syringae were grown for $72 \mathrm{~h}$ as described above. Following the removal of planktonic cells by washing, $100 \mu \mathrm{l}$ of a $10 \mu \mathrm{g}$ peroxidase-linked ConA $\mathrm{ml}^{-1}$ solution (Sigma) was applied to each well and incubated for $15 \mathrm{~min}$. Unbound ConA was removed by three washing steps and the bound enzyme conjugate was visualized after addition of $100 \mu \mathrm{l} 2,2^{\prime}$-azinobis(3-ethylbenzthiazoline-6-sulfonic acid) (ABTS) substrate solution (Sigma). Following an incubation of $15 \mathrm{~min}$, the absorbance was measured at $405 \mathrm{~nm}$. To determine unspecific binding of the lectin, medium without cells was used as control. Wells containing samples without added lectin were treated by the same procedure to detect intrinsic bacterial peroxidase activity. To elucidate the role of levan for its interaction with peroxidase-linked ConA, wells containing $P$. syringae biofilms grown in presence and absence of sucrose were incubated with $50 \mu \mathrm{g}$ levanase for $1 \mathrm{~h}$ prior to the addition of enzyme linked-lectin. At least four parallel samples were run in each experiment, and each experiment was repeated at least twice.

Cultivation of biofilms in flow cells. Biofilms were grown in flow cells with individual channel dimensions of $1 \times 4 \times 40 \mathrm{~mm}$ at $20^{\circ} \mathrm{C}$. The flow system was assembled and prepared as described previously (Møller et al., 1998). The flow-chambers were inoculated by injecting $300 \mu \mathrm{l}$ of a bacterial overnight culture diluted to an $\mathrm{OD}_{600}$ of 0.4 into each flow channel with a $0.5 \mathrm{ml}$ syringe. Upon inoculation, the flow channels were left upside down without flow for $2 \mathrm{~h}$, after which medium was pumped $\left(0 \cdot 2 \mathrm{~mm} \mathrm{~s}^{-1}\right)$ through the channels using a Watson Marlow $205 \mathrm{~S}$ peristaltic pump. Channels were irrigated with FAB minimal medium supplemented with $1 \mathrm{mM}$ glucose or citrate plus $1 \mathrm{mM}$ sucrose, or with 50 -fold diluted Luria-Bertani broth (LB) or King's B medium (KB) (King et al., 1954).

Microscopy and image acquisition. Biofilm structures were thoroughly monitored to compare the phenotypes of the tested strains and mutants of $P$. syringae. CLSM was used to visualize biofilms of PG4180.muc-GFP directly. All other derivatives were monitored by CLSM upon cell staining by injection of $300 \mu$ SYTO 62 (Molecular Probes) into the flow chambers. A Zeiss LSM 510 confocal laser scanning microscope equipped with detectors and filter sets for monitoring GFP, SYTO 62, FITC and TRITC fluorescence was used for all microscopic observations and image acquisitions. Images were obtained using a $\times 63 / 1 \cdot 4$ or a $\times 40 / 1 \cdot 3$ objective. Simulated three-dimensional images and sections were generated by using the IMARIS software package (Bitplane AG).
Lectin staining of biofilms in flow cells. Previously, a screening of all commercially available lectins revealed suitable probes for $P$. syringae glycoconjugate staining (Staudt et al., 2003). To allow for a simultaneous screening of several of these lectins in the same flow cell, we applied a protocol of fixing and embedding the biofilm using polyacrylamide as has been applied for FISH in flow-chamber biofilms (Christensen et al., 2002). However, a lower polyacrylamide concentration $(8 \%)$ was used in the present study. After embedding with polymerizing acrylamide, the biofilm was carefully removed from the flow cell and cut into small pieces, which were each stained with an individual lectin and subsequently washed with distilled water to remove unbound lectin.

Application of fluorescently labelled lectins to flow-chamber biofilms was performed as described by Johnsen and co-workers (Mathee et al., 1999). Briefly, the flow-chamber biofilm was fixed using $4 \% p$ formaldehyde for $1 \mathrm{~h}$, washed and incubated for $30 \mathrm{~min}$ in the presence of the fluorescently labelled lectins (final concn $0 \cdot 1 \mathrm{mg} \mathrm{ml}^{-1}$ ). Finally, the biofilms were washed to remove unbound lectin and cells were stained using $300 \mu \mathrm{l}$ SYTO 62 . FAB medium without carbon source was used for all washing steps. Lectins investigated in more detail were FITC-labelled Concanavalin A (Sigma) and the lectin from Naja mossambica (Sigma), which was labelled with Alexa Fluor 488 using the Alexa Fluor Protein labelling kit (Molecular Probes).

Transcriptional analysis of IscB expression. Plasmid pRLB7.2egfp contains a transcriptional fusion of the $l s c B$ gene from PG4180 with promoterless egfp encoding enhanced green fluorescence protein. To construct this fusion, egfp was excised from pRcCMV3.EGFP (kindly provided by I. B. Leibiger, Karolinska Institute, Sweden) using ApaI and HindIII. The resulting fragment was blunt-ended using Klenow fragment and ligated into XhoIdigested and Klenow-treated plasmid pRLB7.2, which contains a unique $X h o \mathrm{I}$ site inside the $l s c B$ gene. The resulting plasmid was designated pRLB7.2egfp and introduced into PG4180 cells by triparental mating. Transconjugant PG4180 (pRLB7.2egfp) was subsequently grown in liquid FAB medium supplemented with $10 \mathrm{mM}$ glucose and $50 \mathrm{mM}$ sucrose at $20^{\circ} \mathrm{C}$ for $48 \mathrm{~h}$. Likewise, the transconjugant was incubated in wells of polyvinylchloride 96-well microtitre plates at $20^{\circ} \mathrm{C}$ for $96 \mathrm{~h}$. To assess fluorescence during growth of bacteria in liquid culture, aliquots were harvested at distinct optical densities, centrifuged, suspended in PBS ( $\mathrm{pH} \mathrm{7 \cdot 4)}$ ) and adjusted to an OD value of $0 \cdot 5$. To assess fluorescence of attached bacterial cells in microtitre plate wells, the wells were rinsed carefully and filled with PBS ( $\mathrm{pH} \mathrm{7 \cdot 4)}$ prior to fluorescence determination. Parallel wells were stained as described for the microtitre plate biofilm formation assay with crystal violet in order to estimate the amount of attached cells. Fluorescence was quantified using a Dynatech Laboratories Fluorolite-100 microplate reader set to an excitation wavelength of $470 \mathrm{~nm}$ with emission detection at $560 \mathrm{~nm}$.

\section{RESULTS}

\section{Levan and alginate are not required for formation of static biofilms}

EPSs have previously been shown to play a role in attachment and biofilm formation. Consequently, we compared EPSpositive and levan- and/or alginate-deficient derivatives of $P$. syringae for their ability to form biofilms on polystyrene surfaces in wells of microtitre plates using the crystal violet staining method. PG4180.muc, PG4180, PG4180.M6 and PG4180.M6(pBBR3-AXSalgT) all formed surface-associated biofilms under static conditions (Fig. 1). With the exception of biofilms of PG4180.muc which produced slightly more 


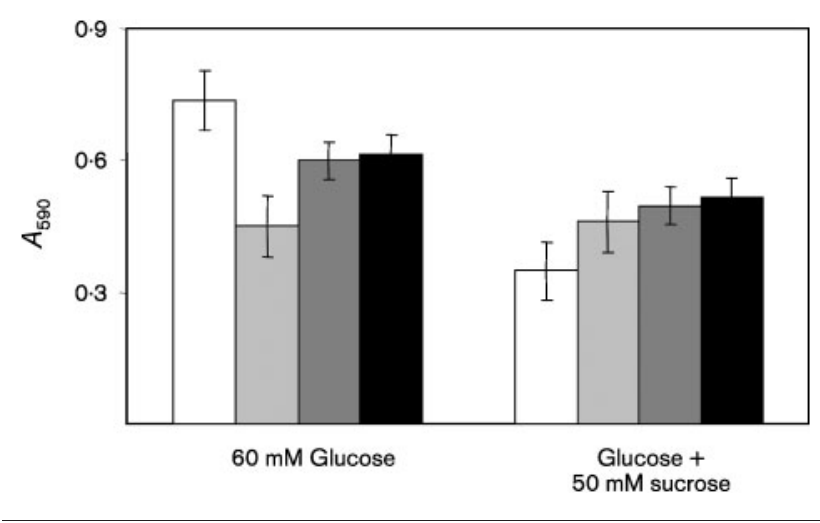

Fig. 1. Biofilm formation of $P$. syringae PG4180.muc (white), PG4180 (light grey), PG4180.M6 (dark grey) and PG4180.M6(pBBR-AXSalgT) (black) in microtitre plates. The strains were grown as surface-attached biofilms in polyvinylchloride microtitre plates using minimal medium supplemented with glucose $(60 \mathrm{mM})$ or glucose $(10 \mathrm{mM})$ plus sucrose $(50 \mathrm{mM})$ for 2 days at $20^{\circ} \mathrm{C}$. After removing the planktonic cells by washing, the biofilm was stained with $0 \cdot 1 \%$ crystal violet. Bars represent the standard deviation between quadruplicates of a representative experiment.

biomass, no substantial differences in the amount of attached biomass could be observed by crystal violet staining. When sucrose was added, PG4180 (levan ${ }^{+}$alginate $^{-}$), PG4180.M6 (levan $^{-}$alginate $^{-}$) and PG4180.M6(pBBR3-AXSalgT) (levan $^{-}$alginate $^{+}$) showed the same phenotype as observed above. However, a considerable decrease in biofilm formation was observed when PG4180.muc (levan ${ }^{+}$alginate $^{+}$) was incubated in the presence of sucrose. Our results suggest that neither levan nor alginate is required for biofilm formation of $P$. syringae in a static system. Interestingly, the presence of sucrose seemed to inhibit biofilm formation when both alginate and levan were produced.

\section{Biofilm development by the levan- and alginate-producing strain PG4180.muc}

Next, the impact of alginate and levan on the development of biofilms in flow chambers was investigated. For this, strain PG4180.muc was tagged with $g f p$. Flow chambers irrigated with FAB medium containing citrate and sucrose were inoculated with exponentially grown cells from a PG4180.muc-GFP culture and biofilm formation was monitored by CLSM over time (Fig. 2). Cells initially formed flat, irregularly shaped microcolonies, which later became ball-shaped microcolonies and towers. In the beginning, microcolonies were predominantly formed at the edges of the flow chamber, i.e. in regions with lower laminar flow. Subsequently, the flow channel was completely covered with microcolonies after about $7 \mathrm{~d}$. Mature biofilms of $P$. syringae showed a heterogeneous appearance and consisted of towers (up to $120 \mu \mathrm{m}$ thick), ballshaped microcolonies, irregularly shaped network-forming microcolonies and substratum-proximal areas with only a few attached cells (Fig. 2).

After 3 days of growth, some of the initially compact microcolonies partially dissolved from the inside due to high cell motility. This resulted in hollow spheres (voids) within ball-shaped microcolonies, towers and network-forming microcolonies (Fig. 2). Numerous motile cells were observed in mature biofilms. Experiments were repeated with untagged PG4180.muc either not stained or stained
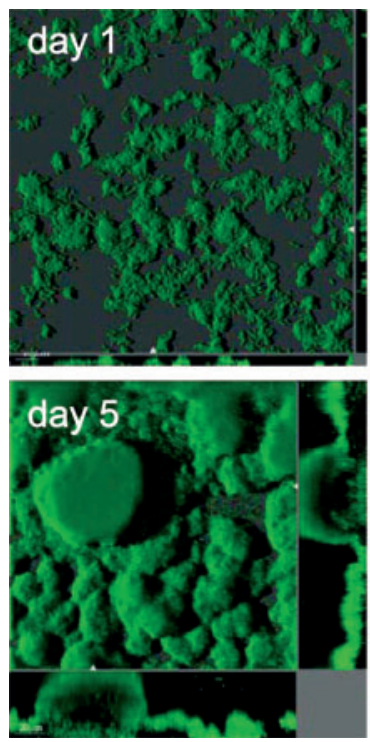
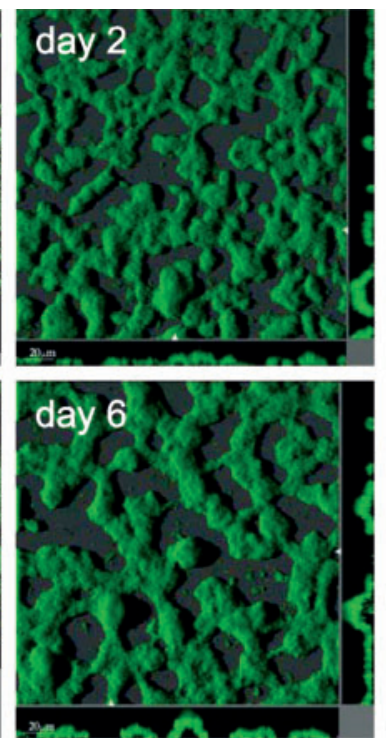
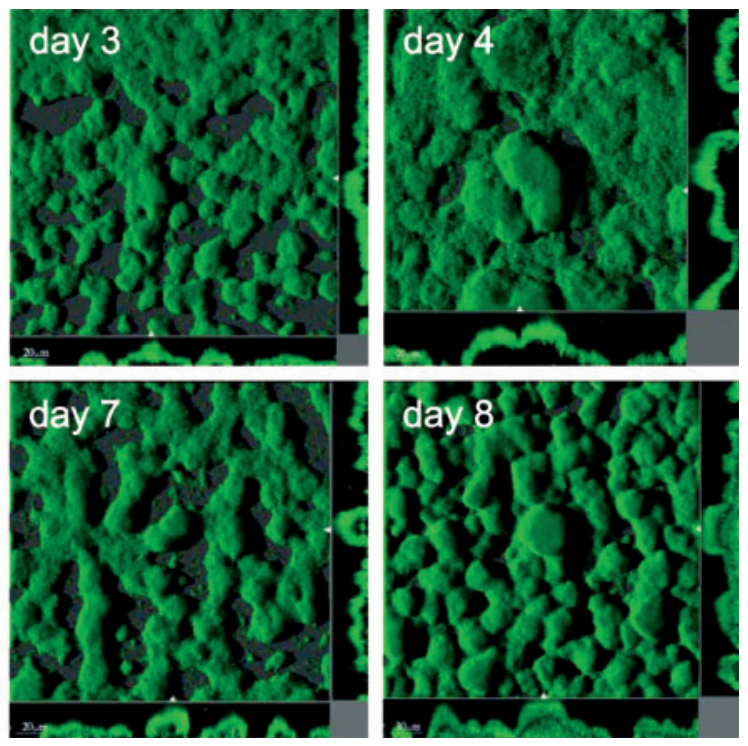

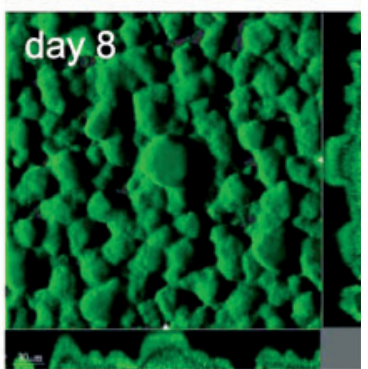

Fig. 2. Biofilm development of $P$. syringae PG4180.muc. A diluted overnight culture of GFP-tagged PG4180.muc-GFP was inoculated into a flow chamber irrigated with minimal medium containing $1 \mathrm{mM}$ citrate and $1 \mathrm{mM}$ sucrose. The development of the flow-chamber biofilm was observed by CSLM for 8 days. 

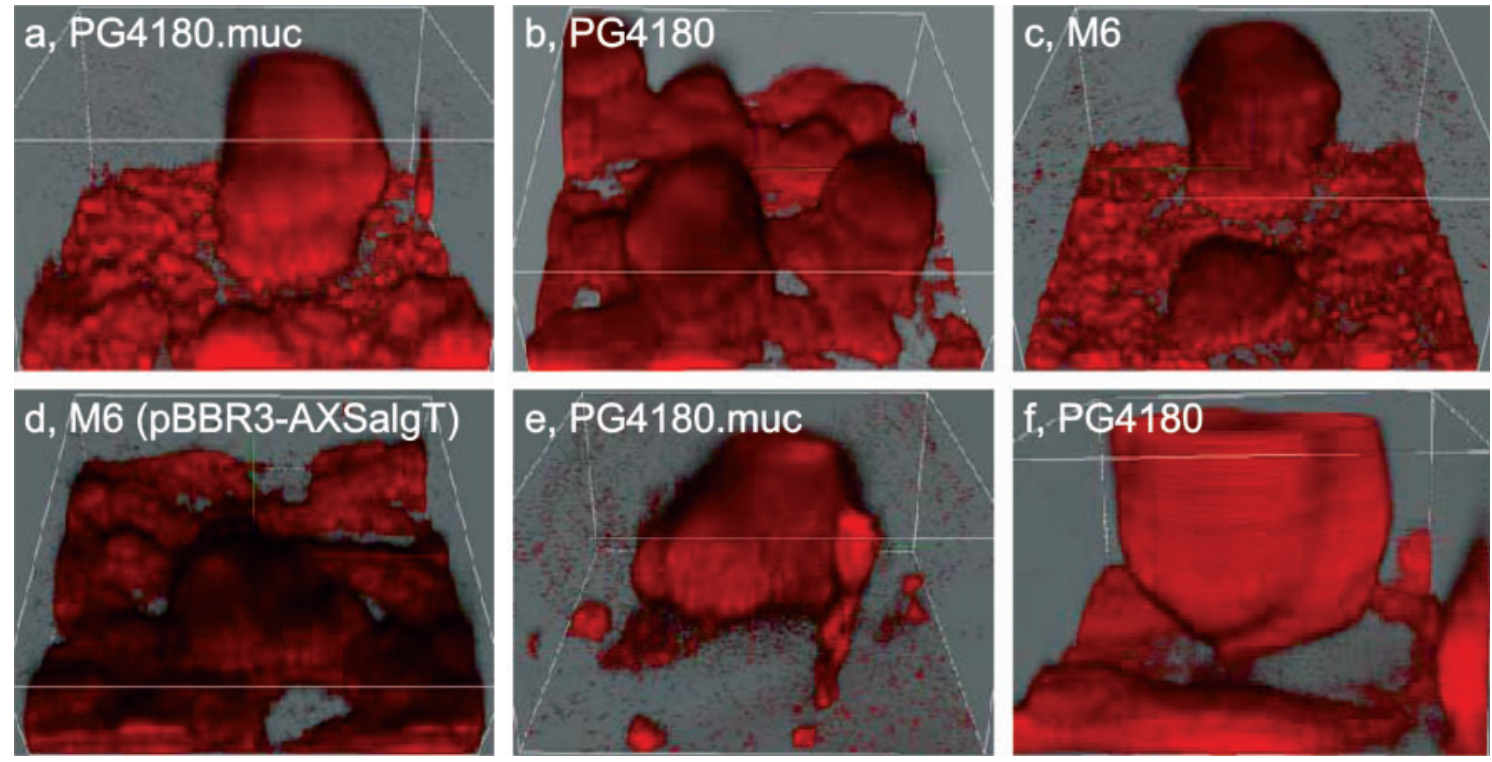

Fig. 3. CLSM side view projections showing the spatial structure of $P$. syringae PG4180.muc, PG4180, PG4180.M6 and PG4180.M6(pBBR-AXSalgT) biofilms. Flow-chamber biofilms were grown in minimal medium supplemented with $1 \mathrm{mM}$ citrate plus $1 \mathrm{mM}$ sucrose. After 9 days, cells were stained by SYTO 62 and representative pictures were taken from different positions of the biofilm close to the inlet. Similar structures, such as dense towers, were found in all four PG4180 derivatives. Mature microcolonies of all four strains dissolved at the substratum, frequently resulting in microcolonies connected to the substratum with only a small stalk (e, f).

with the nucleic acid-binding dye SYTO 62, yielding very similar results.

Biofilms of PG4180.muc-GFP grown in rich medium (LB or KB medium) supplemented with or without sucrose exhibited similarly heterogeneous structures as biofilms grown in minimal medium. However, biofilms developed faster in rich as opposed to minimal medium. After $8 \mathrm{~d}$, the number of cells dispersing from biofilms grown under rich medium conditions was higher than that of the minimal medium control. Consequently, rich-medium biofilms were generally thinner than those grown in minimal medium and consisted of attached cells and small microcolonies at some areas of the substratum. As expected, there were no effects of sugar supplementation in biofilm development in rich medium.

Interestingly, when biofilms of levan-producing PG4180 derivatives were supplemented with sucrose, characteristic blebs of different sizes (about 2-15 $\mu \mathrm{m}$ diam.) developed in mature biofilms (5-8 days). These blebs did not develop under sucrose-deficient conditions. They were clearly distinguishable from air bubbles and thus were probably filled with liquid.

\section{Flow-chamber biofilms of EPS-deficient $P$. syringae}

To investigate the individual impact of the two EPSs on $P$. syringae biofilm structure, PG4180 derivatives deficient in levan or alginate synthesis, or both, were compared with
PG4180.muc (alginate ${ }^{+}$levan $^{+}$). Experimental conditions were made appropriate for levan production by supplementing the cultures with sucrose. Biofilms were stained after 6 and 9 days with SYTO 62 prior to CSLM analysis. All four derivatives formed heterogeneous, dynamic biofilms with similar structures, i.e. dense towers and ballshaped microcolonies (Fig. 3). PG4180.muc and PG4180 biofilms were thicker than those of the levan-deficient derivatives, PG4180.M6 and PG4180.M6(pBBR3-AXSalgT), with about 100 vs $63 \mu \mathrm{m}$ maximum height. Dissolution of the biofilms by cell motility or physical sloughing proceeded regardless of whether or not levan or alginate was formed.

To check the integrity of the EPS phenotypes of the four $P$. syringae derivatives, biofilms were recovered from the flow chambers after $10 \mathrm{~d}$ and grown on $\mathrm{KB}$ or minimal agar plates containing sucrose. Levan synthesis was confirmed by the formation of mucoid colonies on agar plates containing sucrose, and alginate formation by the presence of mucoid colonies on KB agar. Under all conditions, the bacteria isolated from the flow channels continued to exhibit the expected EPS phenotypes.

\section{Specific binding of fluorescently labelled lectins to $P$. syringae flow-chamber biofilms}

To determine the specificity of diverse fluorescently labelled lectins for levan or alginate produced in $P$. syringae biofilms, we tested 64 commercially available lectins for binding to 
PG4180.muc biofilms. The lectins were applied to flowchamber biofilms, which had been fixed and embedded in polyacrylamide. This procedure allowed individual staining of several samples from the same biofilm using each individual lectin. A total of 11 out of the 64 tested fluorescently labelled lectins bound to the PG4180.muc biofilm.

Furthermore, the specificity of the 11 positive lectins was screened in flow-chamber biofilms from all four PG4180 derivatives using CSLM analysis. Surprisingly, none of the 11 lectins was found to bind specifically to the levan-deficient and alginate-producing transconjugant PG4180.M6(pBBR3AXSalgT). In contrast, all but one of the lectins showed no significant difference in dependence of the genotype of the other three tested PG4180 derivatives. Interestingly, FITClabelled ConA did not bind to biofilms of the levan-deficient mutant PG4180.M6, nor its alginate-producing derivative,
PG4180.M6(pBBR-AXSalgT), suggesting that ConA might specifically interact with levan, but not with alginate. Binding of this lectin to biofilms of $P$. syringae was studied in more detail (see below).

\section{Spatial distribution of levan in $P$. syringae biofilms}

Biofilms of levan-producing PG4180.muc and PG4180 were grown in minimal or rich medium supplemented with sucrose as described above, incubated with FITC-labelled ConA and analysed by CLSM. The fluorescently labelled ConA specifically bound to the centre of microcolonies in a condensed form (Fig. 4a, b, c). Interestingly, ConA mainly accumulated in the internal voids of mature microcolonies and in the blebs characteristic of levan production (Fig. 4ad). However, neither all voids nor all microcolonies were stained evenly by ConA (Fig. 4a). Moreover, ConA did not
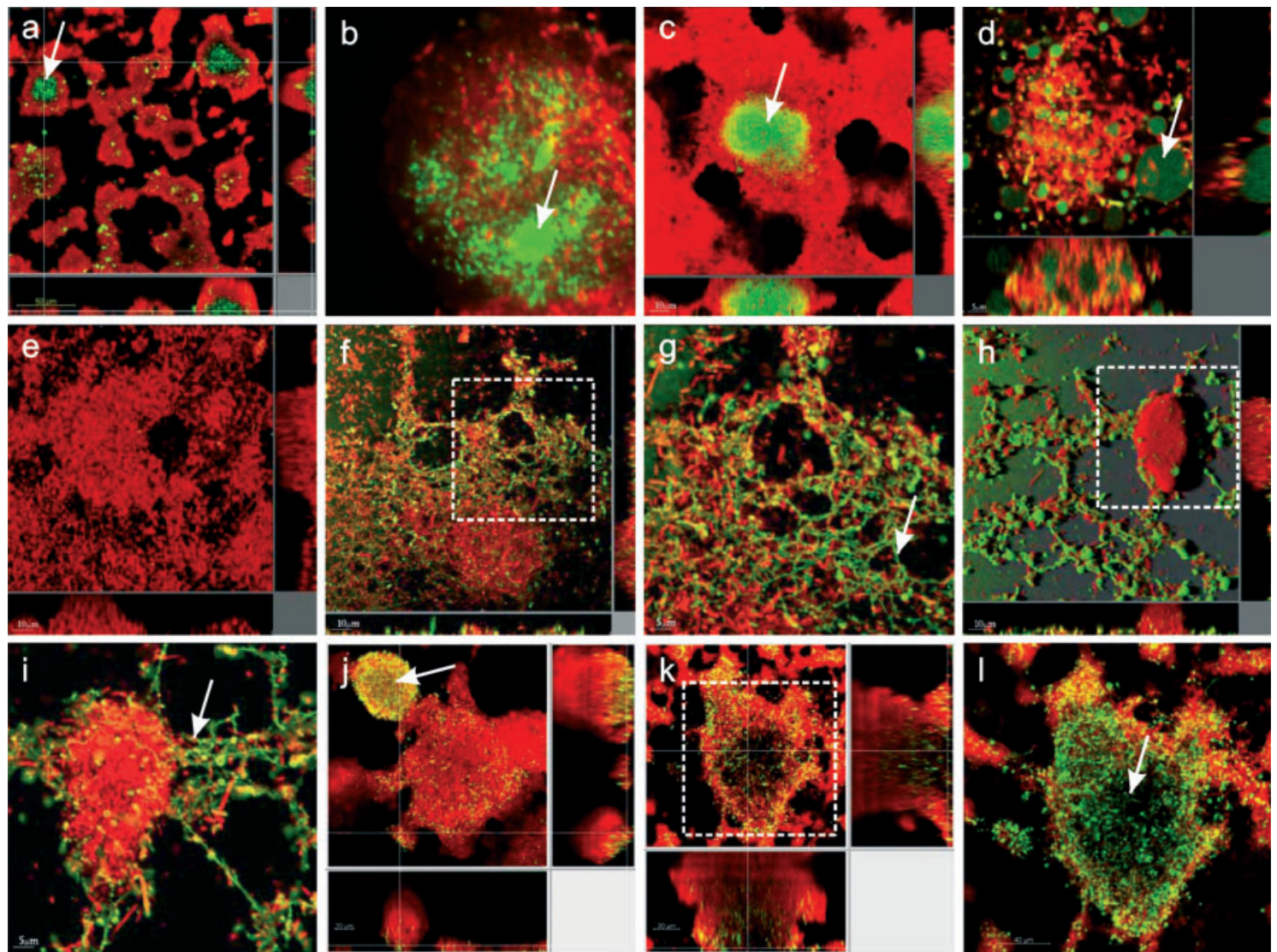

Fig. 4. Binding of lectins to $P$. syringae biofilms. CLSM optical sections of 9 - to 10-day-old biofilms of $P$. syringae PG4180.muc, stained using the fluorescently labelled lectins (green) and SYTO 62 (red). Except for (e) (diluted LB without sucrose) and (j-l) (minimal medium with $1 \mathrm{mM}$ citrate plus $1 \mathrm{mM}$ glucose), all biofilms were grown in minimal medium supplemented with $1 \mathrm{mM}$ citrate plus $1 \mathrm{mM}$ sucrose. (a-d) Binding of FITC-labelled ConA (green) to biofilms of PG4180.muc grown in the presence of sucrose (pictures were taken at different positions of the biofilm close to the inlet); arrows indicate the presence of levan indicated by ConA-binding. ConA did not bind to $P$. syringae PG4180.muc biofilms grown without sucrose (e). Binding of the Alexa 488-labelled lectin from Naja mossambica to P. syringae PG4180.muc biofilms ( $\mathrm{f}-\mathrm{i})$ and to PG4180 biofilms (j-l). Boxes in (f), (h) and (k) indicate positions which are magnified in (g), (i) and (I), respectively. Arrows point to the presence of a third EPS indicated by binding of the Naja lectin. 
significantly bind to biofilm regions consisting of a network of flat microcolonies. Control experiments with SYTO 62 alone gave no signals under the CLSM settings used for FITC-linked ConA. ConA also bound to microcolonies of non-fixed biofilms, suggesting that the fixation step had no influence on the specificity of the binding.

ConA did not bind to biofilms when PG4180.muc and PG4180 were grown in medium lacking sucrose (Fig. 4e). Additionally, no ConA binding could be observed for the levan-deficient mutant PG4180.M6 grown either with or without sucrose (data not shown). In summary, these findings suggested that ConA binds specifically to levan which mainly accumulates in the internal voids of mature microcolonies of $P$. syringae.

To investigate the dynamics of levan production in biofilms, we stained channels of PG4180.muc biofilms at different time points $(3-10 \mathrm{~d})$. No binding of ConA to early-stage biofilms was observed. In contrast, the lectin bound to mature biofilms after about $7 \mathrm{~d}$ growth. As described above, the preferred sites of binding were internal voids of microcolonies and differently sized blebs (Fig. 4a-d). Consequently, the blebs might either consist of or partially contain levan.

\section{Peroxidase-linked ConA binds to levan in microtitre plate biofilms of $\boldsymbol{P}$. syringae}

To further investigate whether ConA binds specifically to levan, we applied an ELLA using peroxidase-linked ConA on 7-day-old biofilms of all four PG4180 derivatives grown in the presence or absence of sucrose in microtitre plates (Fig. 5). Additionally, a transconjugant of mutant PG4180.M6, PG4180.M6(pRLB7.2), complemented for levan production by the introduction of a functional $l s c B$ gene in trans, was used. This transconjugant had initially been tested positive for levan formation on agar plates containing sucrose (data not shown). ConA bound to PG4180.muc, PG4180 and
PG4180.M6(pRLB7.2) biofilms grown in the presence of sucrose. A significantly lower level of binding of ConA was observed when these strains were grown in the absence of sucrose. As expected, biofilms of the levan-deficient controls, PG4180.M6 and PG4180.M6(pBBR-AXSalgT), could not be stained with ConA. Controls involving incubation of $P$. syringae biofilms in the presence of ABTS substrate without prior treatment with peroxidase-linked ConA revealed no interfering endogenous peroxidase activity (data not shown).

Incubation of biofilms of all levan-producing PG4180 derivatives in the presence of levanase (up to $200 \mu \mathrm{g}$ ) prior to the ELLA led to a decrease in the absorption at $405 \mathrm{~nm}$ to the level of the levan-deficient biofilms, whereas the levandeficient mutants did not show a difference in ConA binding after this treatment (Fig. 5).

\section{Temporal analysis of levansucrase gene expression}

To assess the function of void-associated levan accumulation in either structure formation or nutrient storage, respectively, a time study on $l s c B$ gene expression was conducted (Fig. 6). First, this experiment was done in FAB liquid cultures. Transconjugant PG4180(pRLB7.2egfp) containing a transcriptional $l s c B$ : egfp fusion expressed the highest normalized fluorescence levels in the early exponential growth phase with moderately declining levels of reporter gene expression during late exponential and stationary growth phases (Fig. 6a). The plateau of fluorescence in the developing late exponential phase is possibly due to the stability of green fluorescent protein (personal observation, data not shown). This result suggested that levansucrase is mainly produced during the onset of bacterial growth. Next, the experiment was repeated in 96-well microtitre plates under conditions identical to those used for the ELLA. Fluorescence values normalized towards bacterial growth and attachment via crystal violet staining peaked in the early phase of this experiment with slightly

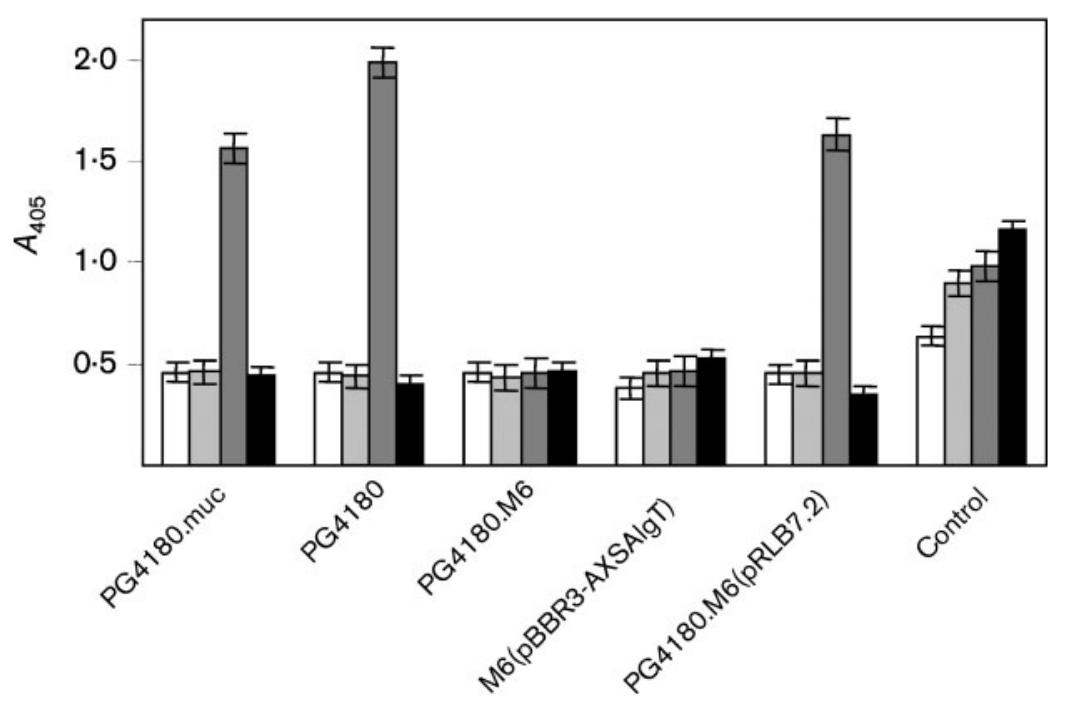

Fig. 5. Binding of peroxidase-linked ConA (ELLA) to microtitre plate biofilms of $P$. syringae. PG4180.muc, PG4180, PG4180.M6, PG4180.M6(pBBR-AXSalgT) and PG4180.M6(pRLB7.2) were grown for 4 days at $20^{\circ} \mathrm{C}$ in wells of microtitre plates in minimal medium containing $60 \mathrm{mM}$ glucose (white bars), $60 \mathrm{mM}$ glucose with $50 \mu \mathrm{g}$ levanase (light grey bars), $10 \mathrm{mM}$ glucose/50 mM sucrose (dark grey bars) or $10 \mathrm{mM}$ glucose/50 mM sucrose with $50 \mu \mathrm{g}$ levanase (black bars) prior to addition of ConA. Controls were carried out without cells inside the wells. Bars represent the standard deviation between quadruplicates of a representative experiment. 


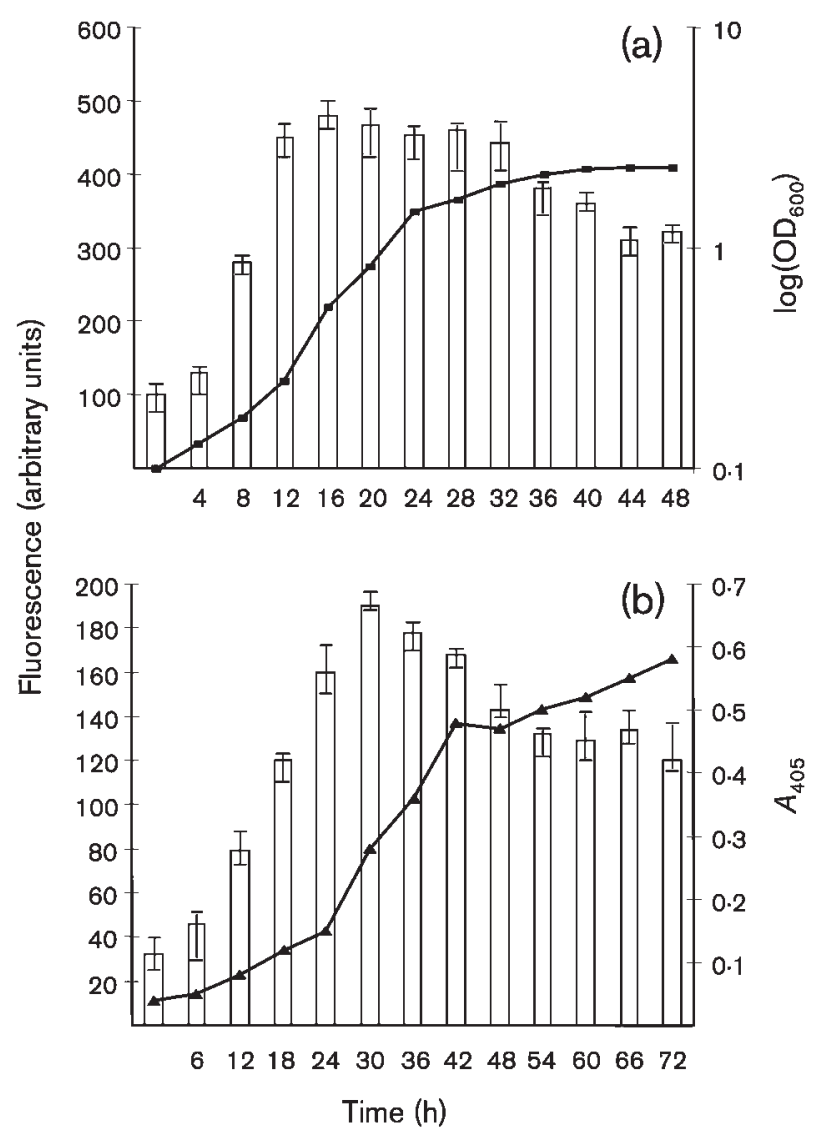

Fig. 6. Transcriptional analysis of $/ s c B$ in liquid cultures (a) and microtitre plates (b). For liquid cultures, PG4180(pRLB7.2egfp) was grown in $F A B$ medium flasks at $20^{\circ} \mathrm{C}$ for $48 \mathrm{~h}$. Fluorescence (normalized towards $\mathrm{OD}_{600}$ ) was determined after harvesting, resuspension of cells in PBS and adjusting suspensions to an $\mathrm{OD}_{600}$ of 0.5 (a). For microtitre plate cultures, the transconjugant was incubated in 96-well microtitre plates at $20^{\circ} \mathrm{C}$ for $96 \mathrm{~h}$. Fluorescence of attached cells (normalized towards crystal violet staining) was determined after rinsing the wells and resuspension in PBS. Parallel wells were stained with crystal violet to estimate the amount of attached cells (b). Bars represent the standard deviation between quadruplicates of a representative experiment.

declining levels in later stages, thus confirming the results from the liquid culture incubations (Fig. 6b). In summary, $l s c B$ is likely to be mainly expressed in the early exponential phase, making a potential quorum-sensing-associated or stationary-phase regulation of this gene rather questionable.

\section{Binding of Naja mossambica lectin reveals the presence of a potential new EPS}

ConA showed specificity to levan, and neither alginate nor levan significantly influenced the structure of the P. syringae biofilms produced. Thus, one of the other positively screened lectins, Alexa-488-labelled lectin from Naja mossambica, was subsequently tested in more detail.
Binding of Naja lectin to mature $P$. syringae biofilms revealed a different binding pattern than ConA. Interestingly, this lectin bound in a web-like manner (Fig. $4 \mathrm{f}-\mathrm{i}$ ). In contrast to ConA, binding of Naja lectin was not detected in the voids of large microcolonies or blebs but was found specifically surrounding dense microcolonies of non-fixed biofilms. Additionally, fibres penetrating the dense microcolonies were stained by Naja lectin (Fig. 4j-1). Naja lectin bound to biofilms of levan-deficient PG4180 derivatives and to those of levan-positive PG4180 strains in the absence of sucrose, suggesting that this lectin was not specific to levan (Fig. 4k-1). Consequently, Naja lectin might stain a yet-tobe-characterized novel EPS or other polymeric structure(s).

\section{DISCUSSION}

EPSs have been shown to play important roles in attachment and structural development of mature biofilms (Sutherland, 2001; Watnick \& Kolter, 1999). Herein, the impact and distribution of levan and alginate in $P$. syringae biofilms was investigated in either static microtitre plate assays or in continuous flow chamber experiments. Surprisingly, neither EPS had a major impact on biofilm formation, suggesting that as-yet-uncharacterized additional polymeric substance(s) mainly contribute to the formation and structure of biofilms.

Fluorescently labelled lectins were applied to study the spatial localization of EPSs, demonstrating for the first time that the lectin ConA specifically binds to levan of $P$. syringae. ConA binding in mature biofilms suggested that levan might accumulate within cell-depleted voids in the centre of microcolonies and in blebs. We ruled out that ConA labelling was due to better accessibility of this lectin to these spaces, since wild-type biofilms grown without sucrose formed the same structures but did not stain with ConA. In support of this hypothesis, ConA only bound to biofilms of cells which produced levansucrase and were supplemented with sucrose. The results were confirmed by applying ELLA to P. syringae biofilms. Moreover, pre-treatment of biofilms of levansucrase-expressing cells grown in the presence of sucrose with levanase reduced the binding of peroxidaselinked ConA to background levels.

The association of ConA binding to levan inside the cell-free voids and blebs of microcolonies allows us to speculate that the voids and blebs may function as reservoirs of carbohydrates. Breakdown of levan chains to fructosyl residues during starvation periods may provide nutrients to the biofilm cells. Thus, levan might act as an energy storage complex for $P$. syringae. This hypothesis is supported by two findings from this study: (i) levan does not contribute to the overall biofilm structure, as shown by the phenotype of levan-deficient strains; and (ii) levansucrase gene expression occurs mainly in the early stages of bacterial growth and biofilm development. Since levansucrase is an extremely stable extracellular protein (Hettwer et al., 1995; Li \& Ullrich, 2001) it might be synthesized during the onset of 
biofilm formation and then might contribute to the successive accumulation of nutrient storage macromolecules inside cell-free areas of the biofilm when sucrose is available. Burne et al. (1996) had suggested a similar function of levan produced by $S$. mutants in the oral cavity.

ConA did not bind to biofilms of the levan-deficient, alginate-producing transconjugant PG4180.M6(pBBR3AXSalgT). This finding clearly ruled out $P$. syringae alginate being bound by ConA, and this is contradictory to results for alginate-containing $P$. aeruginosa biofilms in which ConA was demonstrated to bind to alginate (Strathmann et al., 2002). This discrepancy may be due to the different chemical properties of the alginate(s) produced by either pseudomonad as reflected in different proportions of mannuronic and guluronic acid monomers and different degrees of $\mathrm{O}$ acetylation. Our data give no conclusive information on any particular structure-determining role for alginate in $P$. syringae. It has long been assumed that alginate is the primary secreted EPS in P. aeruginosa biofilms (Evans \& Linker, 1973), but recent data have indicated that alginate is not a significant component of the primary structural matrix of $P$. aeruginosa biofilms (Friedman \& Kolter, 2004a; Hentzer et al., 2001; Jackson et al., 2004; Matsukawa \& Greenberg, 2004; Nivens et al., 2001; Wozniak et al., 2003). Besides structural differences between the EPSs involved in ConA binding for $P$. syringae and $P$. aeruginosa biofilms, respectively, the topological binding pattern was distinct for each organism. In contrast to mature $P$. syringae biofilms, ConA yielded cloud-like cell-containing regions that were heterogeneously distributed throughout $P$. aeruginosa biofilms (Strathmann et al., 2002). When comparing biofilms of both species, it should be noted that the experimental set-up of Wingender et al. (1999) and our study differ significantly.

When analysing the growth-stage dependency of levan production during development of $P$. syringae biofilms, significant ConA binding was only observed in mature 7- to 10-day-old biofilms. In contrast to our findings, timeresolved studies on EPS production in Sphingomonas sp. flow-chamber biofilms using ConA revealed a particular role of EPSs in the initial establishment of the biofilm (Kuehn et al., 2001). Similarly, we used a ConA-specific ELLA with static microtitre plates and found an earlier appearance of levan build-up under those conditions. The latter result was supported by the transcriptional analysis of $l s c B$ for which an association with quorum sensing or stationary-phase dependence remained highly unlikely.

Binding of a Naja mossambica lectin indicated the presence of at least one additional EPS in biofilms of $P$. syringae, regardless of the investigated genotypes. It might stabilize the biofilm structure by forming a dense web of fibres. Consequently, our future experiments will aim to identify the missing EPS(s), which might be fundamental to biofilm formation, and to further dissect the potential role of levan as a long-term nutrient storage source for $P$. syringae.

\section{ACKNOWLEDGEMENTS}

We thank U. Kuhlicke for technical assistance during the lectin screening, and T. Schaefer and P. Rahbek Østergaard for providing levanase. This study was supported by the Deutsche Forschungsgemeinschaft.

\section{REFERENCES}

Arrieta, J., Hernandez, L., Coego, A., Suarez, V., Balmori, E., Menendez, C., Petit-Glatron, M. F., Chambert, R. \& SelmanHousein, G. (1996). Molecular characterization of the levansucrase gene from the endophytic sugarcane bacterium Acetobacter diazotrophicus SRT4. Microbiology 142, 1077-1085.

Boch, J., Joardar, V., Gao, L., Robertson, T. L., Lim, M. \& Kunkel, B. N. (2002). Identification of Pseudomonas syringae pv. tomato genes induced during infection of Arabidopsis thaliana. Mol Microbiol 44, 73-88.

Bogs, J. \& Geider, K. (2000). Molecular analysis of sucrose metabolism of Erwinia amylovora and influence on bacterial virulence. J Bacteriol 182, 5351-5358.

Burne, R. A., Chen, Y. Y., Wexler, D. L., Kuramitsu, H. \& Bowen, W. H. (1996). Cariogenicity of Streptococcus mutans strains with defects in fructan metabolism assessed in a program-fed specific-pathogen-free rat model. J Dent Res 75, 1572-1577.

Christensen, B. B., Haagensen, J. A., Heydorn, A. \& Molin, S. (2002). Metabolic commensalism and competition in a two-species microbial consortium. Appl Environ Microbiol 68, 2495-2502.

Danese, P. N., Pratt, L. A. \& Kolter, R. (2000). EPS production is required for development of Escherichia coli K-12 biofilm architecture. J Bacteriol 182, 3593-3596.

Dedonder, R. (1966). Levansucrase from Bacillus subtilis. Methods Enzymol 8, 500-506.

Evans, L. R. \& Linker, A. (1973). Production and characterization of the slime polysaccharide of Pseudomonas aeruginosa. J Bacteriol 116, 915-924.

Fett, W. F. \& Dunn, M. F. (1989). EPSs produced by phytopathogenic Pseudomonas syringae pathovars in infected leaves of susceptible hosts. Plant Physiol 89, 5-9.

Friedman, L. \& Kolter, R. (2004a). Two genetic loci produce distinct carbohydrate-rich structural components of the Pseudomonas aeruginosa biofilm matrix. J Bacteriol 186, 4457-4465.

Hentzer, M., Teitzel, G. M., Balzer, G. J., Heydorn, A., Molin, S., Givskov, M. \& Parsek, M. R. (2001). Alginate overproduction affects Pseudomonas aeruginosa biofilm structure and function. J Bacteriol 183, 5395-5401.

Hettwer, U., Gross, M. \& Rudolph, K. (1995). Purification and characterization of an extracellular levansucrase from Pseudomonas syringae pv. phaseolicola. J Bacteriol 177, 2834-2839.

Jackson, K. D., Starkey, M., Kremer, S., Parsek, M. R. \& Wozniak, D. J. (2004). Identification of psl, a locus encoding a potential EPS that is essential for Pseudomonas aeruginosa PAO1 biofilm formation. J Bacteriol 186, 4466-4475.

Keane, P. J., Kerr, A. \& New, P. B. (1970). Crown gall of stone fruit. II. Identification and nomenclature of Agrobacterium isolates. Aust J Biol Sci 23, 585-595.

Keith, R. C., Keith, L. M. W., Hernández-Guzmán, G., Uppalapati, S. R. \& Bender, C. L. (2003). Alginate gene expression by Pseudomonas syringae pv. tomato DC3000 in host and non-host plants. Microbiology 149, 1127-1138. 
King, E. O., Ward, M. K. \& Raney, D. E. (1954). Two simple media for the demonstration of pyocyanin and fluorescin. J Lan Clin Med 44, 301-307.

Klausen, M., Heydorn, A., Ragas, P., Lambertsen, L., AaesJorgensen, A., Molin, S. \& Tolker-Nielsen, T. (2003). Biofilm formation by Pseudomonas aeruginosa wild type, flagella and type IV pili mutants. Mol Microbiol 48, 1511-1524.

Koch, B., Jensen, L. E. \& Nybroe, O. (2001). A panel of Tn7-based vectors for insertion of the $g f p$ marker gene or for delivery of cloned DNA into Gram-negative bacteria at a neutral chromosomal site. J Microbiol Methods 45, 187-195.

Kuehn, M., Mehl, M., Hausner, M., Bungartz, H. J. \& Wuertz, S. (2001). Time-resolved study of biofilm architecture and transport processes using experimental and simulation techniques: the role of EPS. Water Sci Technol 43, 143-150.

Lawrence, J. R., Neu, T. R. \& Swerhone, G. D. W. (1998). Application of multiple parameter imaging for the quantification of algal, bacterial and exopolymer components of microbial biofilms. J Microbiol Methods 32, 253-261.

Leriche, V., Sibille, P. \& Carpentier, B. (2000). Use of an enzyme-linked lectinsorbent assay to monitor the shift in polysaccharide composition in bacterial biofilms. Appl Environ Microbiol 66, 1851-1856.

Li, H. \& Ullrich, M. S. (2001). Characterization and mutational analysis of three allelic $l s c$ genes encoding levansucrase in Pseudomonas syringae. J Bacteriol 183, 3282-3292.

Mathee, K., Ciofu, O., Sternberg, C. \& 9 other authors (1999). Mucoid conversion of Pseudomonas aeruginosa by hydrogen peroxide: a mechanism for virulence activation in the cystic fibrosis lung. Microbiology 145, 1349-1357.

Matsukawa, M. \& Greenberg, E. P. (2004). Putative EPS synthesis genes influence Pseudomonas aeruginosa biofilm development. J Bacteriol 186, 4449-4456.

McKenney, D., Hubner, J., Muller, E., Wang, Y., Goldmann, D. A. \& Pier, G. B. (1998). The ica locus of Staphylococcus epidermidis encodes production of the capsular polysaccharide/adhesin. Infect Immun 66, 4711-4720.

Møller, S., Sternberg, C., Andersen, J. B., Christensen, B. B. \& Molin, S. (1998). In situ gene expression in mixed-culture biofilms: evidence of metabolic interactions between community members. Appl Environ Microbiol 64, 721-732.

Neu, T. R. \& Lawrence, J. R. (1999). Lectin-binding analysis in biofilm systems. Methods Enzymol 310, 145-152.

Nivens, D. E., Ohman, D. E., Williams, J. \& Franklin, M. J. (2001). Role of alginate and its $\mathrm{O}$ acetylation in formation of Pseudomonas aeruginosa microcolonies and biofilms. J Bacteriol 183, 1047-1057.

Osman, S. F., Fett, W. F. \& Fishman, M. L. (1986). EPSs of the phytopathogen Pseudomonas syringae pv. glycinea. J Bacteriol 166, 66-71.
O'Toole, G. A. \& Kolter, R. (1998). Initiation of biofilm formation in Pseudomonas fluorescens WCS365 proceeds via multiple, convergent signalling pathways: a genetic analysis. Mol Microbiol 28, 449-461.

Penaloza-Vazquez, A., Kidambi, S. P., Chakrabarty, A. M. \& Bender, C. L. (1997). Characterization of the alginate biosynthetic gene cluster in Pseudomonas syringae pv. syringae. J Bacteriol 179, 4464-4472.

Sato, S., Koga, T. \& Inoue, M. (1984). Isolation and some properties of extracellular D-glucosyltransferases and D-fructosyltransferases from Streptococcus mutans serotypes c, e, and f. Carbohydr Res 134, 293-304.

Staudt, C., Horn, H., Hempel, D. C. \& Neu, T. R. (2003). Screening of lectins for staining lectin-specific glycoconjugates in the EPS of biofilms. In Biofilms in Medicine, Industry and Environmental Biotechnology, pp. 308-326. Edited by P. Lens, A. P. Moran, T. Mahony, P. Stoodley \& V. O'Flaherty. London: IWA Publishing.

Strathmann, M., Wingender, J. \& Flemming, H. C. (2002). Application of fluorescently labelled lectins for the visualization and biochemical characterization of polysaccharides in biofilms of Pseudomonas aeruginosa. J Microbiol Methods 50, 237-248.

Sutherland, I. (2001). Biofilm EPSs: a strong and sticky framework. Microbiology 147, 3-9.

Thomas, V. L., Sanford, B. A., Moreno, R. \& Ramsay, M. A. (1997). Enzyme-linked lectinsorbent assay measures $N$-acetyl-D-glucosamine in matrix of biofilm produced by Staphylococcus epidermidis. Curr Microbiol 35, 249-254.

Watnick, P. I. \& Kolter, R. (1999). Steps in the development of a Vibrio cholerae El Tor biofilm. Mol Microbiol 34, 586-595.

Whitchurch, C. B., Tolker-Nielsen, T., Ragas, P. C. \& Mattick, J. S. (2002). Extracellular DNA required for bacterial biofilm formation. Science 295, 1487.

Wingender, J., Neu, T. R. \& Flemming, H.-C. (1999). Microbial Extracellular Polymeric Substances. Berlin: Springer.

Wolfaardt, G. M., Lawrence, J. R. \& Korber, D. R. (1999). Function of EPS. In Microbial Extracellular Polymeric Substances, pp. 171-200. Edited by J. Wingender, T. R. Neu \& H.-C. Flemming. Berlin: Springer.

Wozniak, D. J., Wyckoff, T. J., Starkey, M., Keyser, R., Azadi, P., O'Toole, G. A. \& Parsek, M. R. (2003). Alginate is not a significant component of the extracellular polysaccharide matrix of PA14 and PAO1 Pseudomonas aeruginosa biofilms. Proc Natl Acad Sci U S A 100, 7907-7912.

Yu, J., Peñaloza-Vázquez, A., Chakrabarty, A. M. \& Bender, C. L. (1999). Involvement of the EPS alginate in the virulence and epiphytic fitness of Pseudomonas syringae pv. syringae. Mol Microbiol 33, 712-720. 